\title{
Neurotoxoplasmosis Diagnosis for HIV-1 Patients by Real-Time PCR of Cerebrospinal Fluid
}

\author{
Fábio Luís Nascimento Nogui ${ }^{1}$, Sandro Mattas ${ }^{2}$, Gilberto Turcato Júnior ${ }^{1}$ and David Salomão Lewi ${ }^{1}$ \\ ${ }^{1}$ Federal University of São Paulo, Division of Infectious Disease; ${ }^{2}$ Federal University of São Paulo, Division of Neurology; São Paulo, SP, \\ Brazil
}

Encephalitis caused by Toxoplasma gondii is the most common cause of central nervous system damage in patients with acquired immunodeficiency syndrome (AIDS). Toxoplasma may infect any of the brain cells, thus leading to non-specific neurotoxoplasmosis clinical manifestations including focused or non-focused signs and symptoms of central nervous system malfunction. Clinical development ranges from insidious display during weeks to experiencing acute general confusion or ultimately fatal onset. Cerebral toxoplasmosis occurs in advanced stages of immunodeficiency, and the absence of anti-toxoplasmosis antibodies by the immunofluorescence method does not allow us to rule out its diagnosis. As specific therapy begins, diagnosis confirmation is sought through clinical and radiological response. There are few accurate diagnosis methods to confirm such cases. We present a method for T. gondii DNA detection by real time PCR-Multiplex. Fifty-one patients were evaluated; 16 patients had AIDS and a presumptive diagnosis for toxoplasmosis, 23 patients were HIV-positive with further morbidities except neurotoxoplasmosis, and 12 subjects were HIV-negative control patients. Real time PCR-Multiplex was applied to these patients' cephalorachidian liquid with a specific $T$. gondii genome sequence from the 529bp fragment. This test is usually carried out within four hours. Test sensitivity, specificity, positive predictive value, and negative predictive value were calculated according to applicable tables. Toxoplasma gondii assay by real time Multiplex of cephalorachidian fluid was positive for 11 out of 16 patients with AIDS and a presumptive diagnosis for cerebral toxoplasmosis, while none of the 35 control patients displayed such a result. Therefore, this method allowed us to achieve $68.8 \%$ sensitivity, $100 \%$ specificity, $100 \%$ positive predictive value, and $87.8 \%$ negative predictive value. Real time PCR on CSF allowed high specificity and good sensitivity among patients who presumably had cerebral toxoplasmosis. Since this is a low invasive method, it could be included in the diagnosis algorithm of patients with AIDS and central nervous system damage.

Key-Words: AIDS, cerebral toxoplasmosis, real time polymerase chain reaction.

Neurological complications occur in $39 \%$ to $70 \%$ of AIDS patients [1-3]. The most frequent opportunistic infections are cerebral toxoplasmosis, cryptococcal meningitis, progressive multifocal leukoencephalopathy (PML), tuberculous meningitis, and cytomegalovirus encephalitis (CMV). In addition to infections, central nervous system primary lymphoma is also an important cause of focused brain damage in such patients. An accurate diagnosis of these neurological complications is crucial, since most such complications are likely to be treated, and prompt effective intervention may eventually yield to longer survival or better quality of living. Most commonly, encephalic toxoplasmosis in AIDS patients is caused by reactivation of a chronic infection. The incidence of cerebral toxoplasmosis in HIV-infected patients is proportional to the prevalence of $T$. gondii latent infection among the population in general. Prior to antiretroviral therapy, one out of three HIV-infected persons could develop encephalic toxoplasmosis with progressive immunological deterioration if no specific prophylaxis were applied $[1,4,5]$. Current USA information, as well as information gathered from other regions, has shown a decrease in brain toxoplamosis

Received on 19 July 2008; revised 7 December 2008.

Address for correspondence: Dr. Fábio Luís Nascimento Nogui. Rua das Camélias, 321 ap 83. Zip code: 04048-060. São Paulo/SP, Brazil. Phone: +5511 5581-1200. Fax: +55 11 5087-9886. E-mail: fnogui@globo.com.

The Brazilian Journal of Infectious Diseases 2009;13(1):18-23. (C) 2009 by The Brazilian Journal of Infectious Diseases and Contexto Publishing. All rights reserved. among AIDS patients as a result of powerful antiretroviral therapy and the use of sulfa derivatives as a primary prophylaxis against Pneumocystis jirovecii pneumonia, which indirectly prevents $T$. gondii infection. Such a decrease evolved from 2.1/100 patients/year in 1992 to $0.7 / 100$ patients/ year in 1997 [6].

In Brazil, there has also been a significant drop in the number of encephalic toxoplasmosis cases within the last years. According to the São Paulo State Epidemiology Surveillance Bulletin, the number of cerebral toxoplasmosis cases fell from 1,408 (17\%) in 1980-1989 to 3,224 (10.1\%) in 2001-2005 [7].

Among immunocompetent patients, toxoplasmosis diagnosis can usually be attained either through direct parasite detection or differences in specific antibody titers in serology tests. In the case of AIDS patients, however, it is recommended to use an algorithm based on imaging examination criteria, i.e. brain CT scan and/or MRI, along with therapeutic proof for around 14 days. Treatment failure usually leads to serious clinical involvement due to misdiagnosis.

We evaluated a quick T.gondii encephalitis diagnosis method using the cephalorachidian liquid of HIV-infected patients by means of real time PCR-Multiplex.

\section{Material and Methods}

Patients

This project was approved by the research and ethics committees of the institutions involved (São Paulo state AIDS/ STD Reference \& Training Center, and the São Paulo Federal 
University Hospital). Fifty-one patients were evaluated, among which 30 were from the São Paulo Federal University Hospital and 21 were from the São Paulo state AIDS/STD Reference \& Training Center). In this group, 16 subjects were HIV-positive with a presumptive diagnosis of cerebral toxoplasmosis, which included signs and symptoms of central nervous system malfunction and mass damage detected by brain CT scan or MRI. Response to specific treatment was exhibited two weeks later. Thirty-five subjects were included as control patients, among which 23 were HIV-infected patients and 12 were nonHIV patients who displayed other diseases or neurological signs.

The assay was targeted at the 529-bp repeat element (GenBank accession numbers AF 487550 and AF 146527), repeated more than 300-fold in the genome of T.gondii (26). Human B-actin gene was co-amplified and detected as the internal control for DNA isolation and PCR amplification. The reactions were performed on the ABI PRISM 7700 Sequence Detection System, ABI PRISM 7500 Sequence Detection System (Applied Biosystems) or Roto-Gene 300 (Corbett Research), using two different TaqMan MGB probes, one for T.gondii 529-bp fragment and one for human B-actin gene. In the sensitivity test, we applied serial T. gondii DNA dilutions. The detection threshold was estimated as $80 \mathrm{fg}$ of DNA, which is equivalent to one parasite per reaction. Negative controls were used in order to achieve higher quality control. The result was shown as detected, and was calculated by interposition of the parasite standard dilution curve. The average duration of this test was four hours.

\section{Statistical Analysis}

Sensitivity, specificity, positive predictive value, and negative predictive value were calculated according to applicable tables. Differences in proportions were compared by the Fischer's exact test. The Kruskal-Wallis test was applied to continuous variables. We applied a $+/$ - one standard deviation to variables in which the average was used. Odds ratios (ORs) with a 95\% confidence interval were calculated for all variables. We considered $p<0.005$ as a significant value. The statistical analysis was carried out with the Statistical Package for the Social Sciences, version 11.5 (SPSS, Chicago, IL, USA).

\section{Results}

Among the 51 patients evaluated from August 2004 to November 2005, 16 displayed AIDS symptoms and had a presumptive diagnosis of cerebral toxoplasmosis. Thirty-five patients were evaluated as control patients, among which 23 were HIV-infected and 12 were HIV-negative.

All patients underwent fluid punction for Toxoplasma gondii DNA assay by real time polymerase chain reaction.

Among the 16 patients who displayed neurological and radiological clinical signs and symptoms of cerebral toxoplasmosis, nine were males $(56 \%)$. In all patients, serum immunoglobulin (IgG and $\mathrm{IgM}$ ) was detected through immunoassay for T. gondii. Brain damage diagnosis was made by neuroimaging examination. All patients underwent a skull CT scan; three patients additionally underwent an MRI.

The therapy applied was a sulfadiazine-pyrimethamine combination, except for a patient for whom clindamycin was prescribed instead of sulfa. As foreseen in the inclusion criteria, there was a response in all evaluated cases. This response consisted of clinical and radiological improvement by the $14^{\text {th }}$ day of therapy. The number of helper T (CD4) lymphocytes ranged from seven to 212 cells $/ \mathrm{mm}^{3}$ (mean 72.75 cells $/ \mathrm{mm}^{3}$; median 44 cells $\left./ \mathrm{mm}^{3}\right)$. In nine patients $(56 \%)$, the number of CD4 T-cells was lower than 100 cells $/ \mathrm{mm}^{3}$.

In five among the 16 patients with a presumptive diagnosis for cerebral toxoplasmosis who showed a response to treatment, it was not possible to detect cerebral toxoplasmosis DNA by real time PCR. In these patients, the CD4 T-cell count ranged from 9 to 169 cells $/ \mathrm{mm}^{3}$ (mean 98.2 cells $/ \mathrm{mm}^{3}$; median 150 cells $/ \mathrm{mm}^{3}$ ). When we applied the Kruskall-Wallis test, we found no significant difference between the two groups $(p=0.125)$ in the CD4 cell counts (Table 1$)$.

The CSF collection date varied from the $1^{\text {st }}$ to the $9^{\text {th }}$ day following therapy initiation. In 12 cases (75\%), collection was carried out within the first 72 hours of the patient's admission to the hospital.

Some clinical and laboratory variables were compared in the five false negative patients and the 11 patients who gave better results in positive real time PCR. When we considered the difference in proportion between $T$. gondii-positive and negative PCR patients, we found the following: Fever(OR, 0.250; 95\% CI 0.03 - 2.32; $\mathrm{p}=0.299)$, headache (OR, 1.250; 95\% CI 0.146 - 10.699; $\mathrm{p}=1.00$ ), seizures (OR, 0.15;95\% CI0.01 - 1.562; $\mathrm{p}=0.245$ ), motor deficit (OR, 0.56; 95\% CI $0.06-4.75 ; \mathrm{p}=1.00)$, and altered consciousness (OR, 2.62; 95\% CI $0.30-22.99$; $p=0.596)$. Thus, we found no significant differences when we compared the clinical symptoms of the two groups (Table 2).

We worked with two control groups. The first group comprised 23 HIV-positive patients who underwent fluid examination because they were likely to have other neuroinfections than neurotoxoplasmosis. This age group ranged from 27 to 60 years (mean $=39.9$ years); $13(56 \%)$ were males and 10 were females. Of these patients, five $(22 \%)$ were diagnosed with neurosyphilis, four (17\%) with neurocryptococcosis, four $(17 \%)$ with tuberculous meningoencephalitis, two $(9.5 \%)$ were reported to have cephalea symptoms, two (9.5\%) displayed seizures, and the others were found to have hepatic enchephalopathy, bacterial meningitis, progressive multifocal leukoencephalopathy (PML), and chorioretinitis caused by toxoplasmosis with a cerebral vascular accident. One AIDS patient underwent CSF collection to fight acute lymphocytic leukemia through intrathecal chemotherapy.

All 12 non-HIV patients had been admitted to the São Paulo Hospital. The age group ranged from 18 to 75 years (mean $=39.4$ years); eight of the 12 were males and four were females. The neurological diseases that led to CSF collection 
Table 1. Mean and median number of T CD4 lymphocytes among patients with presumptive diagnosis for cerebral toxoplasmosis demonstrated by real -time PCR

\begin{tabular}{lccc}
\hline & Lymphocytes TCD4 $\left(\right.$ cells $\left./ \mathbf{m m}^{\mathbf{3}}\right)$ & \\
\hline PCR(No.) & Mean & Median & p \\
\hline PCR detection & 72.75 & 44 & 0.125 \\
PCR Negative & 98.20 & 150 & \\
\hline
\end{tabular}

Table 2. Clinical features among patients with cerebral toxoplasmosis with positive and negative PCR

\begin{tabular}{lcccccc}
\hline Signs and symptoms & $\begin{array}{c}\text { Cerebral Toxoplasmosis } \\
\text { PCR+(11) }\end{array}$ & & $\begin{array}{c}\text { Cerebral Toxoplasmosis } \\
\text { PCR-(5) }\end{array}$ & p \\
\cline { 2 - 3 } \cline { 5 - 6 } & $\begin{array}{c}\text { No. of } \\
\text { patients }\end{array}$ & $(\%)$ & & $\begin{array}{c}\text { No. of } \\
\text { patients }\end{array}$ & $(\%)$ \\
\hline Abnormal level of consciousness & 7 & 63.6 & & 02 & 40 & 0.596 \\
Headache & 5 & 45.5 & & 2 & 40 & 1.00 \\
Motor weakness & 5 & 45.5 & & 2 & 40 & 1.00 \\
Fever & 3 & 27.3 & & 3 & 60 & 0.299 \\
Seizure & 2 & 18.2 & & 3 & 60 & 0.245 \\
Cranial nerve abnormalities & 2 & 18.2 & & 0 & 0 & 1.00 \\
\hline
\end{tabular}

were as follows: four due to herpetic meningoencephalitis, and the others due to politraumatism, meningioma, cerebral venous thrombosis, Guillain-Barré syndrome, optic neuritis, pineal tumor, chorioretinitis caused by toxoplasmosis, and viral meningitis.

All HIV-positive control subjects yielded negative real time PCR results, showing that the specificity of this method was $100 \%$ in this sample. Patients with chorioretinitisprovokedtomoxoplasmosis (both HIV-positive and HIVnegative) showed negative results in the real time PCR $T$. gondii assay.

Assessment of sensitivity, specificity, positive predictive value, and negative predictive value were calculated in a $2 \times 2$ table. The following results were obtained:

Sensitivity: $68.8 \%$

$[95 \% \mathrm{CI}]$

Specificity: $100.0 \%$

$[44.4 \% ; 85.8 \%$ ]

$[90.4 \% ; 100.0 \%]$

Negative Predictive Value: $87.8 \% \quad[74.5 \% ; 94.7 \%]$

Positive Predictive Value: $100.0 \% \quad[74.1 \% ; 100.0 \%]$

There were no false positives, which confirms the specificity of as high as $100 \%$. However, five cases of patients with cerebral toxoplasmosis and negative real-time PCR were found, resulting in a sensitivity of $68.8 \%$.

\section{Discussion}

Neurological complications often occur in HIV-infected people, and T. gondii encephalitis is the most frequent cause of focal brain disease in AIDS patients [11]. More than $50 \%$ of HIV-positive individuals will eventually develop neurological symptoms of this disease. Such complications are generally observed in advanced stages of the disease, with severe immunodepression. However, $10-20 \%$ of HIV-positive patients may develop opportunistic infections even if there is no immunological failure [12]. Among our cases, only one patient with a cerebral toxoplasmosis diagnosis had T-helper CD4 lymphocyte counts greater than 200 cells $/ \mathrm{mm}^{3}$.

In such neurologically-affected patients, focal brain damage (FBD) features among the main issues while reaching a diagnosis in HIV-1 infected patients. Cerebral toxoplasmosis is the most common cause of FBD among these individuals, though differential diagnoses have also reported lymphomas, TB, and cryptococcosis. According to the latest Epidemiological Bulletin issued by the São Paulo State STD/ AIDS Program in 2005, 20,059 (14.9\%) cases of neurotoxoplasmosis have been reported in Brazil since 1980. Toxoplasma gondii infection may be diagnosed either indirectly through serological methods, or directly through such methods as PCR, hybridization, and histology. Whenever clinical manifestations suggest that there might be brain damage, neuroimaging studies, such as skull CT scan or MRI should be performed. Positive presumptive diagnosis, which is broadly regarded as a suitable clinical practice for HIV patients and is adopted as a rule in both national and foreign hospitals, is based on the patient's clinical conditions, imaging study (CT scan/MRI), and response to treatment after two weeks [13]. This standard diagnosis usually does not consider brain biopsy, which is regarded as the ultimate definite diagnosis, in spite of the risk to which patients are then exposed. A retrospective multicentric study carried out by Antinori with 160 brain biopsies in HIV patients with focal brain damage showed a sensitivity of as high as $87 \%$. Nevertheless, this study led to significant morbidity and mortality (7.5 and 3.1\%, respectively). The causes of such morbidity and mortality were basically due to biopsy-related 
surgical brain hemorrhage, permanent facial-brachial paralysis, transitory neurological failure, surgical wound infection, and osteomyelitis. Biopsy-related mortality occurred, on average, 14 days following surgery and was also related to the stereotaxic method [14].

Moreover, if toxoplasmosis empirical therapy does not lead to clinical improvement, it will eventually delay diagnosis of other possible neuropathologies in these patients, thus affecting prognosis and therapeutic success directly. We evaluated a quick, accurate method for diagnosing cerebral toxoplasmosis so patients do not need to undergo unnecessary treatment and invasive diagnostic procedures. We found that real time PCR of CSF is a relatively simple and quick method that can be performed in developing countries to confirm suspected cases. It is a less invasive procedure when compared with brain biopsy. As opposed to conventional PCR, real time PCR provides a lower false positive risk [15], since there is no technical need to open the sample tubes when amplification is completed [16].

Other studies have also shown that conventional PCR on CSF results in a sensitivity of from $12 \%$ to $70 \%$ (usually $50 \%$ to $60 \%$ ) and a specificity of nearly $100 \%$ in patients with cerebral toxoplasmosis [17-19]. Most studies have demonstrated the diagnostic role of PCR in blood samples, although sensitivity has varied within a broad range, i.e. 25 $77 \%[9,20-22]$.

A retrospective study performed by Antinori analyzed $T$. gondii DNA presence in five HIV-infected patients who were believed to have encephalic toxoplasmosis (Group 1), eight HIV infected patients with other symptoms (Group 2), and seven non-HIV patients with other neurological diseases (Group 3). PCR was positive for two of four of the patients with an ultimate diagnosis of encephalic toxoplasmosis, whereas it was negative in the other groups. This small study confirmed the low sensitivity and high specificity of PCR when applied to encephalic toxoplasmosis diagnosis [14].

Another study analyzed $88 \mathrm{CSF}$ samples from HIV-positive patients, among which 56 had focal brain damage. The patients were prospectively tested for $T$. gondii B1 gene nested PCR. Six out of the 18 patients with cerebral toxoplasmosis (but no patients with other brain disorders) showed a positive PCR result, resulting in $33 \%$ sensitivity and $100 \%$ specificity. This study also proved that early collection leads to higher sensitivity. As the study considered solely encephalic toxoplasmosis patients whose CSF was collected prior to or during the first week of antitoxoplamosis therapy, sensitivity increased to $50 \%$. Antitoxoplamosis prophylaxis had no effect on PCR results [23].

Vidal et al. have recently evaluated CSF samples from 12 AIDS patients with a first episode of cerebral toxoplasmosis, and 18 AIDS patients with other neurological opportunistic diseases and no previous cerebral toxoplasmosis. This evaluation was carried out at the Emilio Ribas Infectology Institute. Samples from all cerebral toxoplasmosis patients showed positive conventional PCR results (sensitivity, 100\%), and the sample from one of the 18 AIDS patients with other neurological diseases also showed positive PCR results $(94.4 \%$ specificity) [24].

In our study, we evaluated HIV-positive individuals who attended the São Paulo Hospital emergency room as well as patients who were admitted to the São Paulo State AIDS/STD Reference \& Training Center with signs and symptoms that suggested focal brain damage. These patients underwent neuroradiological examination and were admitted to the hospital due to suspected encephalic toxoplasmosis. Fluid punction was performed soon after admission. The presumptive diagnosis of these 16 patients was then confirmed as prescribed by the Centers for Disease Control and Prevention [25].

We applied real time PCR based on 200 to 300-fold amplification of the $T$. gondii DNA 529bp fragment. This fragment was selected not only because it is the least repeated one, but also because it bears a more diverging sequence standard, thus causing sensitivity to be greater than that resulting from using the B1 gene [26,27].

Our group of patients with a presumptive diagnosis of neurotoxoplasmosis displayed the typical signs and symptoms of HIV-infected patients, particularly altered consciousness (56.3\%), motor deficit (50\%), and headache (43.8\%). However, no such symptoms prevailed in the group with cerebral toxoplasmosis when compared with patients with other infections. We found no statistical difference related to cerebral toxoplasmosis clinical conditions between PCR positive patients and false negative patients.

As mentioned above, serum immunoglobulin (IgG and IgM) assay for $T$. gondii immunoassay in patients with a presumptive diagnosis was positive for all subjects, which confirms that this group had previously been exposed to the protozoa. Skull CT scan (both with and without contrast) and/ or MRI showed a lesion compatible with cerebral toxoplasmosis (skull CT was performed in 100\% of the patients, while skull MRI was performed in $18.75 \%$ of patients). The prevailing lesion found on neuroimaging examination was related to contrasted annular granuloma.

The prescribed therapy was a sulfadiazine-pyrimethamine combination. We considered a definite diagnosis of cerebral toxoplasmosis as we observed patients' clinical and imaging improvement 14 days following specific therapy initiation.

We found $68.8 \%$ sensitivity, 100\% specificity, 100\% positive predictive value, and $87.8 \%$ negative predictive value. Gianotti et al. applied PCR (either conventional or real time) for $T$. gondii in 52 patients with a presumptive diagnosis of cerebral toxoplasmosis; they achieved 100\% specificity, $16 \%$ sensitivity, $100 \%$ positive predictive value, and $40 \%$ negative predictive value [28].

Cingolani et al. found $33.3 \%$ sensitivity in a study carried out on 88 HIV-1 infected patients, by applying nested PCR. Another study, which also applied nested PCR showed 100\% sensitivity because CSF was collected during the first week of therapy [29]. 
When we reviewed the low sensitivity attained among our toxoplasmosis patients, we found that T-helper/CD4+ lymphocyte counts ranged from 7 to 212 cells $/ \mathrm{mm}^{3}$ (mean 51.7 cells $/ \mathrm{mm}^{3}$, median 27 cells $/ \mathrm{mm}^{3}$ ) in the 11 patients with real time PCR detection, whereas T-helper/CD4+ counts ranged from 9 to 171 cells $/ \mathrm{mm}^{3}$ (mean 98.2 cells $/ \mathrm{mm}^{3}$, median 150 cells/ $\mathrm{mm}^{3}$ ) in patients with a presumptive diagnosis of cerebral toxoplasmosis but no real time PCR detection. Even though the mean and median T CD4 counts in the real time PCR group was lower, such a gap was not found to be significant. Thus, false negative PCR tests would not be justified by this parameter.

Another factor that could otherwise distinguish undetected PCR patients was the amount of brain lesions seen on neuroimaging examination of such patients, since four of 10 showed a single lesion. We could therefore assume that the low parasite load upon fluid punction (notwithstanding the signs and symptoms compatible with cerebral toxoplasmosis) eventually affected $T$. gondii DNA amplification adversely in these patients, who coincidentally showed improved immunity (CD4 counts greater than 100 cells $/ \mathrm{mm}^{3}$ ). This may have had an effect on sensitivity in our sample.

For ethical reasons, we were unable to carry out brain biopsy in patients with a presumptive diagnosis of neurotoxoplasmosis in order to reach a definite etiological diagnosis. However, there was no positive result for real time PCR among control patients, which ultimately confirms the excellent test specificity (100\%). Therefore, finding a positive real time PCR test for T. gondii in CSF is of great diagnostic relevance, particularly in patients whose CT scan or MRI does not appear to be typical. In this case, one would have better reasons as to whether or not carry out such an invasive procedure as a CNS biopsy.

Given the small sample size in our study, we believe that it should be enhanced by further investigation with a greater number of individuals. Carrying out real time PCR in AIDS patients with a suspected diagnosis of cerebral toxoplasmosis may soon become a powerful diagnostic tool, thus preventing patients from undergoing such a high morbidity-mortality examination as brain biopsy. Performing $T$. gondii assay by means of a less invasive method that delivers quicker results such as real time PCR would certainly be a very useful tool in the disease algorithm.

\section{Acknowledgments}

Fábio L. N. Nogui was supported by the Coordenação de Aperfeiçoamento de pessoal de Nível Superior (CAPES) do Ministério da Educação do Brazil. The authors wish to thanks Dr. Mauro Figueredo and Dra. Kozue Myashiro at Fleury Medicine and Health, São Paulo, SP for techical support.

\section{References}

1. Luft B.J., Remington J.S.Toxoplasmic encephalitis in AIDS. Clin Infect Dis 1992;15:211-22.
2. Porter S.B., Sande M.A. Toxoplasmosis of the Central Nervous System in the Acquired Immunodeficiency Syndrome. N Engl J Med 1992;327:1643-8.

3. Levy R.M., Bredesen D.E., Rosenblum M.L. Neurological complications of the acquired immunodeficiency syndrome (AIDS): Experience at UCSF and review of the literature. J Neurosurg 1985;62:475-95.

4. Grant I.H., Gold J.W., Rosemblum M., et al. Toxoplasma gondii serology in HIV-infected patients: the development of central nervous system toxoplasmosis in AIDS. AIDS 1990;4(6):519-21.

5. Zangerle R., Allerbreger F., Pohl P., et al. High risk of develoing toxoplasmis encephalitis in AIDS patients seropositive to Toxoplasma gondii. Med Microbilo Immunol 1991;180(2):59-66.

6. Jones J.L., Hanson D.L., Dworkin M.S., et al. Surveillance for AIDS-defining opportunistic illnesses, 1992 - 1997. MMWR CDC Surveill Summ 1999;48(2):1-22.

7. Boletim Epidemiológico - C.R.T. DST/AIDS Ano XXIV, Número $1,2005$.

8. Johnson J.D., Butcher P.D., Savva D., Holliman R.E. Application of the polymerase chain reaction to the diagnosis of human toxoplasmosis. J Infect 1993;26:147-58.

9. Guy E.C., Joyson D.H. Potential of polymerase chain reaction in the diagnosis of active toxoplasma infection by detection of parasite in blood. J Infect Dis 1995;172:319-22.

10. Montoya J.G. Laboratory diagnosis of Toxoplasma gondii infection and toxoplasmosis. J Infect Dis 2002;185(suppl1):S73-82.

11. Gabudza D.H., Hirsch M.S. Neurologic manifestations of infection with human immunodeficiency vírus: Clinical features and pathogenesis. Ann Intern Med 1987;107:383-91.

12. Bensalem MK, Berger JR. HIV and the Central Nervous System. Comp Ther 2002;28(1):23-33.

13. Subauste CS. Toxoplasmosis and HIV 2006. In: HIV InSite Knowledge Base. 2006.

14. Antinori A., Ammassari A., Luzzati R., et al. Role of brain biopsy in the management of focal lesions in HIV-infected patients. Neurology 2000;995-7.

15. Menotti J., Vilela G., Romand S., et al. Comparison of PCREnzime-Linked Immunosorbent Assay and Real-Time PCR Assay for Diagnosis of an Unusual Case of Cerebral Toxoplasmosis in a Stem Cell Transplant Recipient. J Clin Microbiol 2003;41:5313-6.

16. Costa J.M., Pautas C., Ernault P., et al. Real-Time PCR for diagnosis and follow-up of toxoplasma reactivation after allogeneic stem cell transplantation using fluorescence resonance energy transfer hybridization probe. J Clin Microbiol 2000;38:2929-32.

17. Parmley S.F., Goebel F.D., Remington J.S. Detection of Toxoplasma gondii in cerebrospinal fluid from AIDS patients by polymerase chain reaction. $J$ Clin Microbiol 1992;30(11):3000-2.

18. Dupon M., Cazenave J., Pellegrin J.M., et al. Detection of Toxoplasma gondii by PCR and tissue culture in cerebrospinal fuid and blood of HIV-seropositive patients. J Clin Microbiol 1995;33:2421-6.

19. Eggers C., Gross U., Klinker H., et al. Limited value of cerebrospinal fluid for direct detection of Toxoplasma gondii in toxoplasmic encephalitis associated with in AIDS. J Neurol 1995;242(10):644-9.

20. Lin M., Chen T., Kuo T., Tseng C. Real-time PCR for quantitative detection of Toxoplasma gondii. J Clin Microbiol 2000;38:4121-5.

21. Burg J.L., Grover C.M., Pouletty P. Direct and sensitive detection of a pathogenic protozoan, Toxoplasma gondii by PCR. J Clin Microbiol 1989;27:1782-7.

22. Franzen C., Altfeld M., Hegener P., et al. Limited value of PCR for detection of Toxoplasma gondii in blood from human immunodeficiency virus-infected patients. J Clin Microbiol 1997;35:2639-41. 
23. Skiest D.J. Focal Neurological Disease in Patients with Acquired Immunodeficiency Syndrome. Clin Infect Dis 2002;34:103-13.

24. Vidal J.E., Colombo F.A., Oliveira A.C.P., et al. PCR assay using cerebrospinal fluid for diagnosis of cerebral toxoplasmosis in Brazilian AIDS patients. J Clin Microbiol 2004;42.

25. Center for Disease Control: Revision of surveillance case definition for acquired immunodeficiency syndrome. MMWR 1987;36(suppl 1):1-15.

26. Homan W.L., Vercammen M., De Braekeleer J., Verschueren H. Identification of a 200 - to 300 -fold repetitive $529 \mathrm{bp}$ DNA fragment in Toxoplasma gondii, and its use for diagnostic and quantitative PCR. Int J of Parasitology 2000;30:69-75.
27. Reischl U., Bretagne S., Krüger D., et al. Comparasion of two DNA targets for the diagnosis of Toxoplasmosis by real-time PCR using fluorescence resonance energy transfer hybridization probes. BMC Infectious Diseases $2003 ; 3$.

28. Gianotti N., Cinque P., Castagna A., et al. Diagnosis of toxoplasmic encephalitis in HIV-infected patients. AIDS 1997;11(12):1529-30.

29. Novati R., Castagna A., Morsica G., et al. Polymerase chain reaction for Toxoplasma gondii DNA in cerebrospinal fluid of AIDS patients with focal brain lesions. AIDS 1994;8:1691-4. 